Rafidain Journal of Science

DOI

Vol. 29, No. 3, pp. 61-72, 2020

: 10.33899/rjs.2020.166313

https://rsci.mosuljournals.com

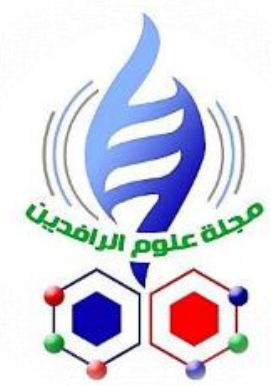

ISSN: 1608-9391

e-ISSN: 2664-2786

Received:28/5/2020

Accepted:10/7/2020

\section{Multicore Photonic Crystal Fibres Designing for Multiplexers and Demultiplexers Applications}

\author{
Miami Mohammed \\ ${ }^{1}$ Institute of Applied Physics/ University of Muenster/ Corrensstrße 2-4, \\ 48149 Muenster/ Germany \\ ${ }^{2}$ Department of Physics/College of Science/ University of Al- \\ Mustansiriyah/ Baghdad/Iraq \\ Ahmad K. Ahmad * \\ Department of Physics/College of Science/ Al-Nahrain University/ \\ Baghdad/Iraq \\ *E-mail: ahmad.kamal@sc.nahrainuniv.edu.iq
}

\begin{abstract}
Coupling properties are significantly affective when the three cores of a photonic crystal fiber are identical or non-identical, we analyze this numerically using COMSOL Multiphysics software 5.5 to gain insight into the nature of the coupling between these cores. A non-identical core produced by a slight mismatch in the propagation constants which are sufficient to suppress coupling between the cores or may strengthen the coupling between the cores because they sometimes have equal propagation constants. By manipulating the central core diameter (increase or decrease) compared to the diameter of the other cores can limit the strength of the coupling between the cores, the coupling efficiency and the coupling length of the cores become decreasing. Introducing anisotropy in three core diameters resulting in the modes of these cores are isolated in a certain region of the array of photonic crystal fiber, as a result, the coupling is suppressed between the modes of cores at the wavelength $1064 \mathrm{~nm}$ while some coupling appears between modes of the cores in the wavelength $1550 \mathrm{~nm}$ where the coupling coefficient is evaluated as a function of wavelength. The coupling between three cores reveals novel characteristics more than two cores and this useful for Multiplexers and Demultiplexers applications
\end{abstract}

Keywords: PCF, Coupling length, Strength of the coupling, Multiplexer, Demultiplexer. 


\section{INTRODUCTION}

During the past decade, multicore fibers (MCFs) designs have been extensively used in optical communication systems. These designs offer a large mode area that is designed to support the propagation of supermodes between cores. This is useful in many applications such as fiber lasers and amplifiers, fiber couplers, fiber sensors, fiber endoscopes, microwave photonics, the mode-division multiplexing (MDM) (Jollivet et al., 2014; Tan et al., 2015; Ahmad et al., 2020). Also, routing and coupling devices depended on multimode interference, the evanescent coupling between cores or waveguides that is important in integrated photonic circuits, optical modulators, mode converters, multiplexers, demultiplexers and switches for high-speed communication, data processing, and integrated quantum operations (Mrejen, et al., 2015; Li et al., 2010). In identical cores PCF (Photonic Crystal Fibres), there is no mismatch between the cores, and therefore, the differential group delay spread (DGDS) becomes zero, this is important for applications such as multiple-input-multiple-output (MIMO) (Wu et al., 2015; Parto et al., 2016). A non-identical core produces by introducing a small change in the core diameters; the resulting small mismatch is enough to reduce the crosstalk between cores and consequently decouple them (Szostklewicz et al., 2016), is possible to achieve demultiplexing in space-division-multiplexed (SDM) using MCFs (Parto et al., 2016). Over the years, there has been much research on interesting to study multicore PCF coupling and decoupling to tailor field between cores in theoretical and experimental, such as (Wu et al., 2015; Yan et al., 2008), some studies have investigated the linear and nonlinear guiding dynamics when three- core PCF with a planer (Yan et al., 2008) and triangular the structures as in (Li et al., 2010).

This paper numerically investigate identical and non-identical three-core photonic crystal fibres (PCFs) by using COMSOL Multiphysics software 5.5, based on the finite element method (FEM). The distributions modal of the supermodes and the coupling lengths of the cores are evaluated. A slight change in the central core diameter is compared to the outer cores affect the strength of coupling between the three cores, further, we describe how to introduce anisotropy in the diameters of all the cores. This produces a small mismatch between the neighboring cores and the cores become decoupled and isolated in a certain region in the array of the PCF. Changing the wavelength has a strong effect on coupling properties between cores even if it is completely different, this study is useful for mode divided multiplexers and demultiplexers applications.

\section{Design methodology and simulation results:}

Design identical and non-identical cores three-core photonic crystal fiber (PCFs) that are placed close to one another, then energy transmission can occur between the three cores through the coupling in their evanescent fields (Mohammed, 2019). We manipulated by changing the central core diameter $3.5 \mu \mathrm{m}$ or $3.2 \mu \mathrm{m}$ or $3.8 \mu \mathrm{m}$ relative to the diameter to other cores $3.5 \mu \mathrm{m}$ to obtain identical and non-identical cores. This design of three-core PCF with structural parameters, such as the hole pitch $\Lambda=4 \mu \mathrm{m}$, hole diameter $\mathrm{d}=1.16 \mu \mathrm{m}$, the air-filling fraction is $\mathrm{d} / \Lambda=0.29$, and core separation $\mathrm{D}=2.5 \mu \mathrm{m}$ at the wavelength $\lambda=1550 \mathrm{~nm}$. The refractive index of the core material $n_{\text {co }}=1.45$ is slightly larger than the refractive index of the cladding material, $n_{\text {clad }}=1.4$ modelled to the light guiding by total internal reflection mechanism (MTIR) between the core and the cladding region. This design is modelled using COMSOL MULTIPHYSICS software 5.5, based on finite element method (FEM) see Fig. (1). FEM allows us to divide the cross-section of threecore PCF into finite small elements by using mesh-free triangular and chose the module of electromagnetic waves-frequency domain to study mode analysis. Supermode analysis of three-core PCF by FEM and it directly solves the Maxwell equations to obtain an approximate value of the effective refractive indexes of the modes, and according to the supermode theory that relies on the mode coupling, there are six modes with different propagation constants either three modes are even and have the same phase or three modes are odd and have phase differences is $\pi$ in both x- and $y$-polarization fields. 

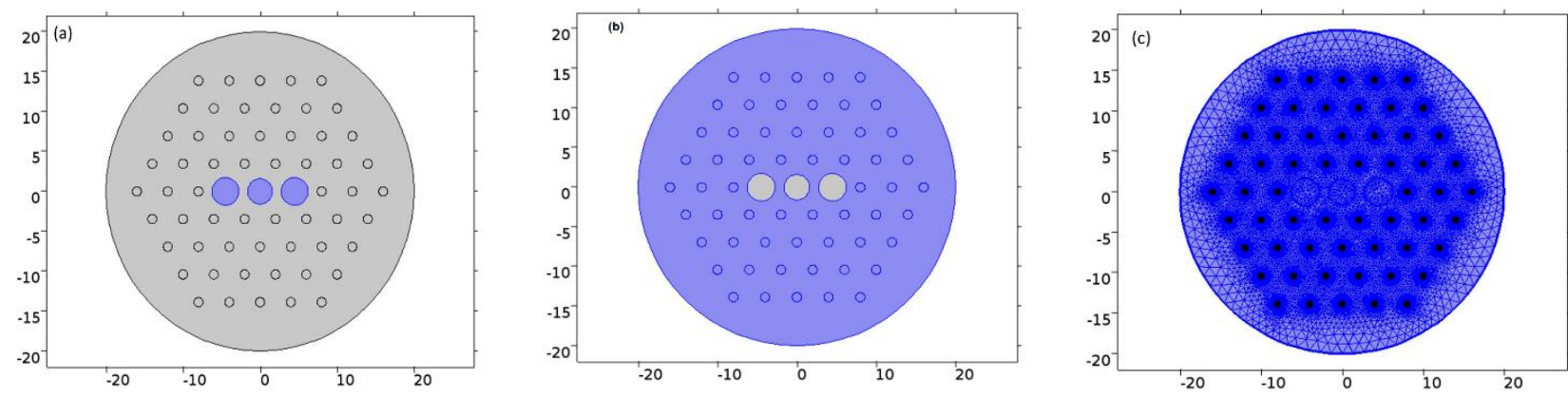

Fig. 1: Geometry structure of three-core photonic crystal fiber with three-ring hexagonal lattice: (a) insert core material; (b) insert the cladding material, (c) mesh free-triangular.

After knowing the effective refractive indexes of the modes, we can directly calculate the coupling length $\left(\mathrm{L}_{\mathrm{c}}\right)$, it defines as a single power is exchanged between three cores, resulting in the weak overlap of the adjacent electric field of three cores. Therefore, a part of the light is confined into one core, then transfer to the other cores after propagation distance known coupling length can be calculated in equation (1) (Mohammed, 2019):

$$
L_{c}=\frac{\pi}{\beta_{\text {even }}-\beta_{\text {odd }}}=\frac{\lambda}{2\left(n_{\text {even }}-n_{\text {odd }}\right)}
$$

$\beta_{\text {even }}$ and $\beta_{\text {odd }}$ represent the difference in propagation constants of even and odd modes, the refractive indexes that correspond to it are ( $n_{\text {even }}$ and $n_{\text {odd }}$ ), as well as the wavelength $\lambda$ that is used in the design. The coupling coefficient $\kappa$ describes the percentage of power transferred between the three cores of the PCF, and $\kappa$ relates to coupling length given by the following relation: $\kappa=\pi / 2 L_{c}$.

The numerical results showed that the coupling length $\mathrm{L}_{\mathrm{c}}$ for the identical core of $3.5 \mu \mathrm{m}$ and the non-identical cores of $3.2 \mu \mathrm{m}$ and $3.8 \mu \mathrm{m}$, the effective mode indexes along the $\mathrm{x}$-polarized field are for the even and odd modes, the differences in the effective refractive index for even and odd modes and the coupling coefficients at the $\lambda=1550 \mathrm{~nm}$ are shown in (Table 1). Fig. (2) represents the relationship between the coupling lengths, the coupling coefficients, and the core diameter for three structures with different designs that may be identical and non-identical in its cores, and this shown in (a) and (b) according to the results shown in (Table 1). These results indicate the possibility of designing a useful system for coupling or splitter with very short coupling lengths in $(\mu \mathrm{m})$ useful in optical communications.

Table 1: Represents the numerical calculation results for comparing three structural designs, one of which is an identical core of $3.5 \mu \mathrm{m}$ and two non-identical cores of $3.2 \mu \mathrm{m}$ and $3.8 \mu \mathrm{m}$ at a wavelength of $1550 \mathrm{~nm}$.

\begin{tabular}{|l|l|l|l|l|l|}
\hline $\begin{array}{c}\text { Core } \\
\text { diameter }\end{array}$ & $\begin{array}{c}\text { Even } \\
\text { mode }\end{array}$ & $\begin{array}{c}\text { Odd } \\
\text { mode }\end{array}$ & $\begin{array}{c}\text { The difference in the } \\
\text { effective refractive index } \\
\text { for even and odd modes }\end{array}$ & $\begin{array}{c}\text { Coupling } \\
\text { length } \\
(\boldsymbol{\mu m})\end{array}$ & $\begin{array}{c}\text { Coupling coefficient } \\
(\boldsymbol{\mu m})^{-\mathbf{1}}\end{array}$ \\
\hline $3.2 \mu \mathrm{m}$ & 1.4299 & 1.4265 & 0.0034 & 227.94 & 0.0068 \\
\hline $3.5 \mu \mathrm{m}$ & 1.4308 & 1.4296 & 0.0012 & 645.83 & 0.0024 \\
\hline $3.8 \mu \mathrm{m}$ & 1.4324 & 1.4297 & 0.0027 & 287.03 & 0.0054 \\
\hline
\end{tabular}



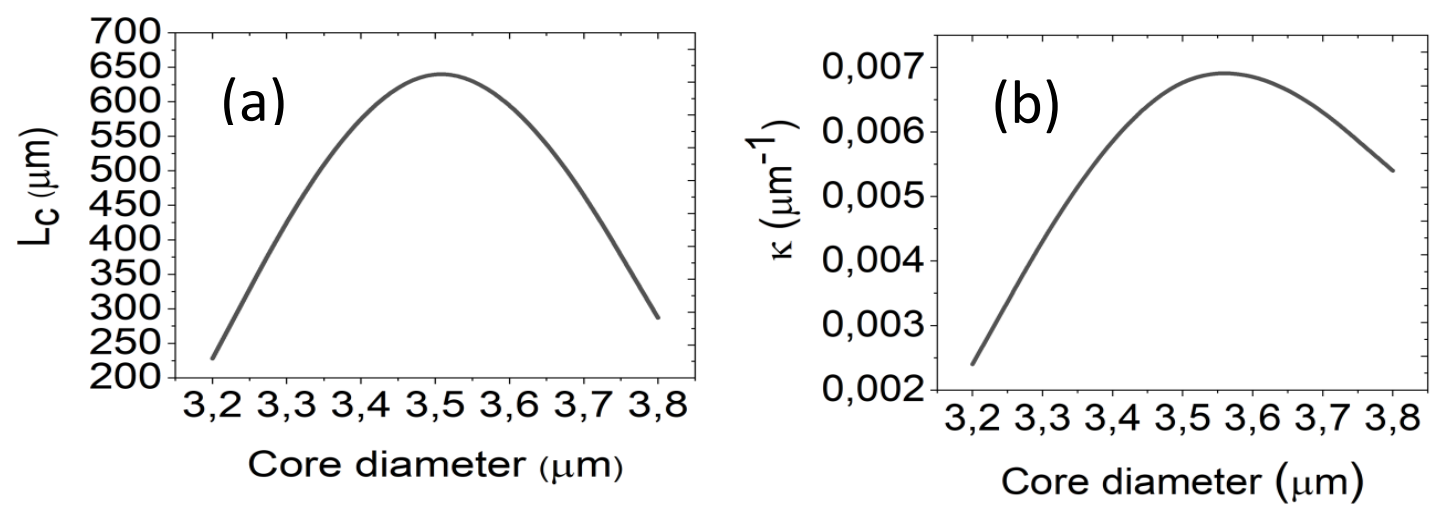

Fig. 2: (a) The coupling length $\mathrm{L}_{C}$ (b) The coupling coefficient $\kappa$, as a function of core diameter at wavelength $1550 \mathrm{~nm}$.

\section{Result of identical and non-identical three cores PCF coupler}

The rate of power transfer between the three cores PCF is known as the coupling length or beat length; consequently, the coupling length and the coupling coefficient are employed to determine the strength of that coupling that depends on the interactions between cores (Reichenbach et al., 2005; 2007).

Supermode is a linear combination of the LP modes of each core, and the total number of nondegenerations supermodes is equivalent to the number $\mathrm{N}$ of cores in the system (Szostklewicz et al., 2016; Tan et al., 2015; Reichenbach et al., 2005; 2007), where the mode of multicore fibers with the different propagation constants contains $2 \mathrm{~N}$ modes, and the factor of two represents two polarization states of modes that referred to as the fundamental modes. The distribution of energy within these individual cores for each of the modes is nearly Gaussian with azimuthal symmetry. The mode solutions are of both even and odd modes for each polarization where the modes will propagate at little different speeds along the PCFs. A linear combination of the LP supermodes of each core with specific phase relation to the electric field distribution of three cores PCF is similar to the single-core fiber where the 1 st supermode has considered the fundamental mode $\mathrm{LP}_{01}$ mode that has the largest effective index is considered as lower-order mode while the supermode $\mathrm{LP}_{11}$ and $\mathrm{LP}_{02}$ are considered as a higher-order mode ( Tan et al., 2015; Kishi et al., 1989; Yu et al., 2006).

Three single power distributions of the six fundamental modes with polarized modes $\mathrm{LP}_{02}$, (show the coupling between the central core and the surrounding cores), $\mathrm{LP}_{11}$ (the strong coupling between the surrounding cores than the central core), $\mathrm{LP}_{01}$ (suppress the coupling between the surrounding cores) for identical cores with diameters $(3.5 \mu \mathrm{m})$, display in Fig. $3 \mathrm{c}$ and non- identical cores with diameters of $3.2 \mu \mathrm{m}$ and $3.8 \mu \mathrm{m}$ with outer core diameters are $(3.5 \mu \mathrm{m})$, are displayed in Fig. $4 c$ and $5 c$, when the light is initially interred to the central core. In $\mathrm{LP}_{01}$ mode, all cores have the same power and intensity, leading to all excited modes within the central core area. In $\mathrm{LP}_{02}$ mode, all the surrounding cores have propagation constants are nearly equal to the propagation constant of the central core. In $\mathrm{LP}_{11}$ mode, the surrounding cores are excited relative to the central core and the strong coupling between them because they have the same propagation constants nearly, therefore, power is limited in the outer cores.

Fig. ( 3 a \& b) show the effective mode index and Surface for Electric field distributions $(\mathrm{V} / \mathrm{m})$ of the x component for identical three cores PCF coupler for even and odd modes for a core diameter of $3.5 \mu \mathrm{m}$. From the numerical results in Fig. (3c) that represent the field distributions for identical line three-core PCF coupler design are $\mathrm{LP}_{02}, \mathrm{LP}_{02}$ and $\mathrm{LP}_{11}$ with the refractive indices are $1.4308,1.4296$ and 1.4282 . The field distributions of identical three cores PCF shows supermodes with the field distributions of $\mathrm{LP}_{02}$ and $\mathrm{LP}_{11}$ that have a nonzero and zero fields in the center core respectively. 
First, concerning $\mathrm{LP}_{02}$ modes, all the outer cores have propagation constants which are converted and almost equal to the propagation constant of the central core, where the mode field is more confined to the centre core. Therefore, the field distributions of $\mathrm{LP}_{02}$ have lower propagation constants owing to the presence of the field in the center core, which means that the power is limited in the central core as shown in Fig. $(3 \mathrm{c})$. Second, the fields distribution of $\mathrm{LP}_{11}$ that have zero fields in the center core, when outer cores (higher-order modes) are excited relative to the central core and coupling between the surrounding cores become strong because of the propagation constants of these cores are equal with each other where the difference between the propagation constants of these cores is zero. The coupling strength normally depended on the mode; this means, the power is limited in the outer core. While Fig. (3d) represents the evolution of the power flow for the fundamental modes of the three cores shown in (c), where each core supports two propagation modes, and the initial input into the central core is at $1.55 \mu \mathrm{m}$. In this Figure, we show six lines of colors, the yellow line represents one of the central core modes, while the pink line represents the other, and the four rest of the colors represent the modes of the cores adjacent to the central core that is represented in light blue, red, green and blue. We notice one mode of the central core (yellow color) is coupled with two modes of the two adjacent cores (green and blue), or the two adjacent cores are strongly coupled together with their modes (light blue and red) without coupled to the mode of the central core or we may find the other mode of the central core (pink) is a weak coupling with the two adjacent cores

Fig. (4) shown the effective mode index and Surface for Electric field distributions $(\mathrm{V} / \mathrm{m})$ of the $\mathrm{x}$ component for non-identical three cores PCF coupler for even and odd modes as in ( $\mathrm{a}$ and $\mathrm{b}$ ) that is represented with a core diameter of $3.2 \mu \mathrm{m}$. Fig. (4c) represents the field distributions for both cases of non-identical three cores PCF are $\mathrm{LP}_{01}, \mathrm{LP}_{02}$ and $\mathrm{LP}_{11}$ with the refractive indices are $1.4299,1.4295$ and 1.4265 . Firstly, concerning $\mathrm{LP}_{01}$ modes, all three cores have the same power where the intensity is equal among all cores and leads to all excited modes within the central core area. Secondly, concerning $\mathrm{LP}_{02}$ modes, all the outer cores have propagation constants which are converted and almost equal to the propagation constant of the central core, where the mode field is more confined to the center core. Therefore, the field distributions of $\mathrm{LP}_{01}$ and $\mathrm{LP}_{02}$ have lower propagation constants owing to the presence of the field in the center core, which means that the power is limited in the central core. Thirdly, the fields distribution of $\mathrm{LP}_{11}$ that have zero fields in the center core, when outer cores (higher-order modes) are excited relative to the central core and coupling between the surrounding cores become strong because of the propagation constants of theses cores are equal with each other where the difference between the propagation constants of these cores is zero and the coupling strength is morally depended on the mode, this means, the power is limited in the outer core. Fig. $(4 \mathrm{~d})$ expresses the evolution of the power flow for the three cores fundamental modes that are presented in (c), where each core supports two propagation modes when initially input into the central core is at $1.55 \mu \mathrm{m}$. We showed six color lines; the yellow line represents one of the central core modes coupled with two modes of adjacent cores (light blue and red), while the pink line represents the other mode of the central core, which not coupled to modes of adjacent cores. The other modes of the adjacent cores (green and blue) are strongly coupled together without coupled to the central core.

Fig. (5) shows the effective mode index and Surface for Electric field distributions $(\mathrm{V} / \mathrm{m})$ of the $\mathrm{x}$ component for non-identical three cores PCF coupler for even and odd modes as in (a \& b) that is represented with a core diameter of $3.8 \mu \mathrm{m}$. Fig. $(5 \mathrm{c})$ represent the field distributions for both cases of non-identical three cores PCF are $\mathrm{LP}_{02}, \mathrm{LP}_{02}$ and $\mathrm{LP}_{11}$ with the refractive indices are 1.4324, 1.4297 and 1.4288. Fig. (5d) represent the evolution of the power flow for the three cores fundamental modes that are presented in (c), the yellow and pink lines that represented the two modes of the central core either a strong or a weak coupling with two modes of adjacent cores as (blue and green) or the other modes of the adjacent cores (light blue and red ) are strongly coupled together without coupled to the central core. 

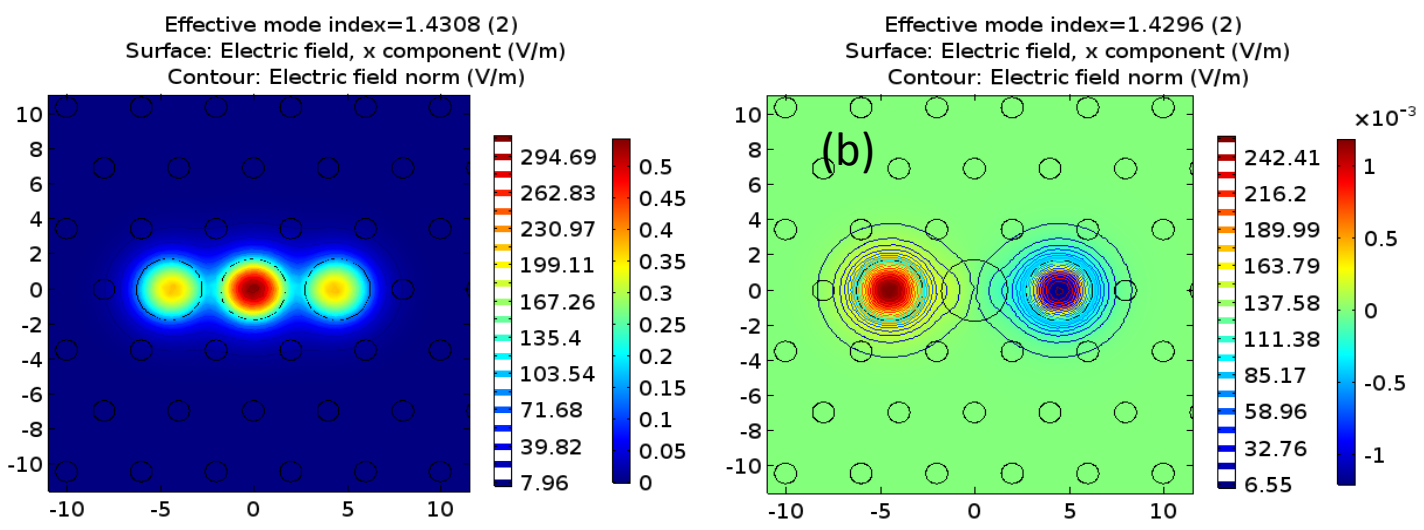

(c)
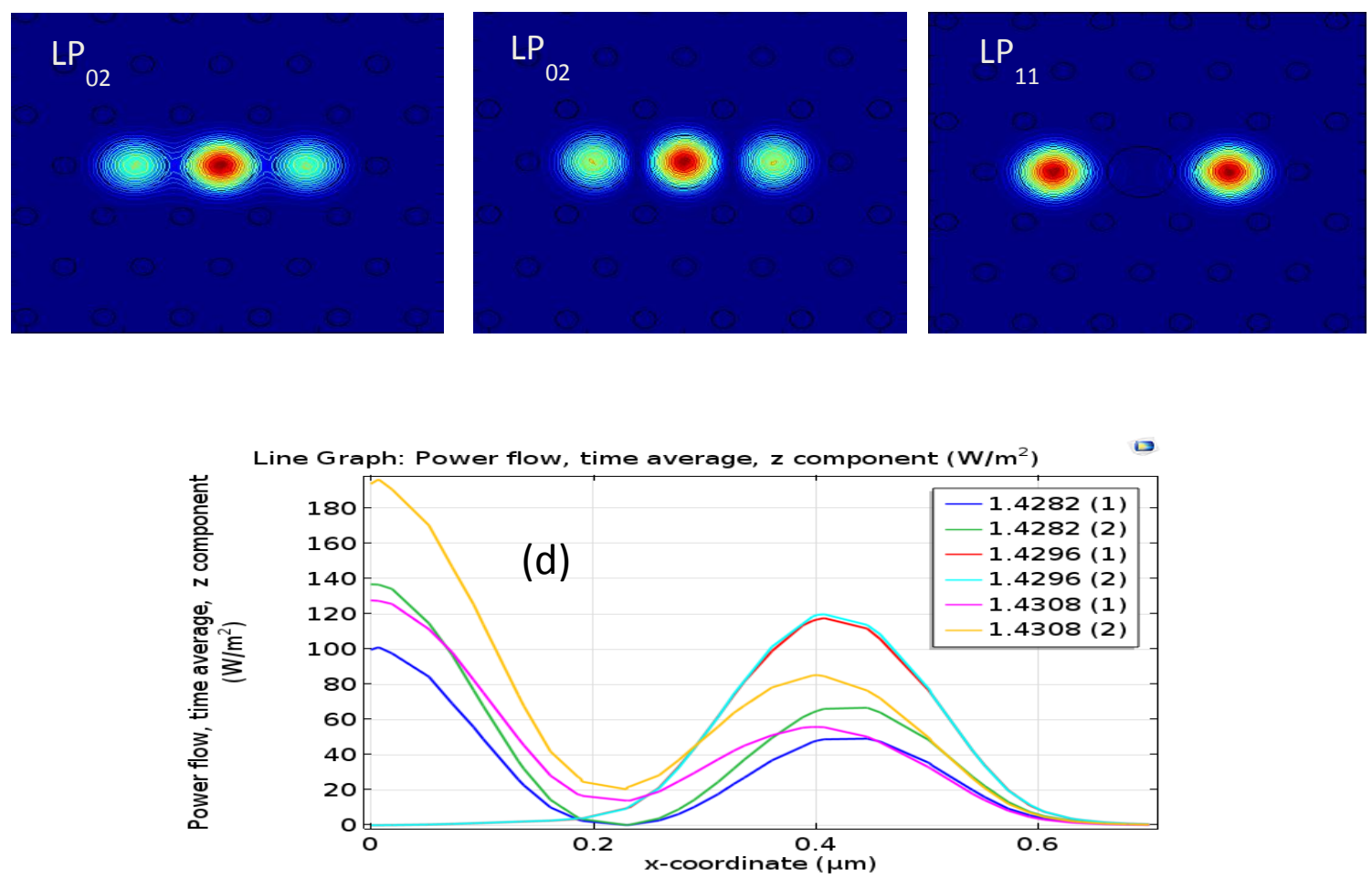

Fig. 3: Effective mode index and Surface Electric field distributions of the $x$ component for (a) Identical and (b) Non-identical, three cores PCF. (c) Power flow distributions of the six fundamental modes of the identical three-core PCF for core diameter 3.5 $\mu \mathrm{m}$. (d) Power flow for the fundamental modes of the three cores at wavelength $1.55 \mu \mathrm{m}$. 

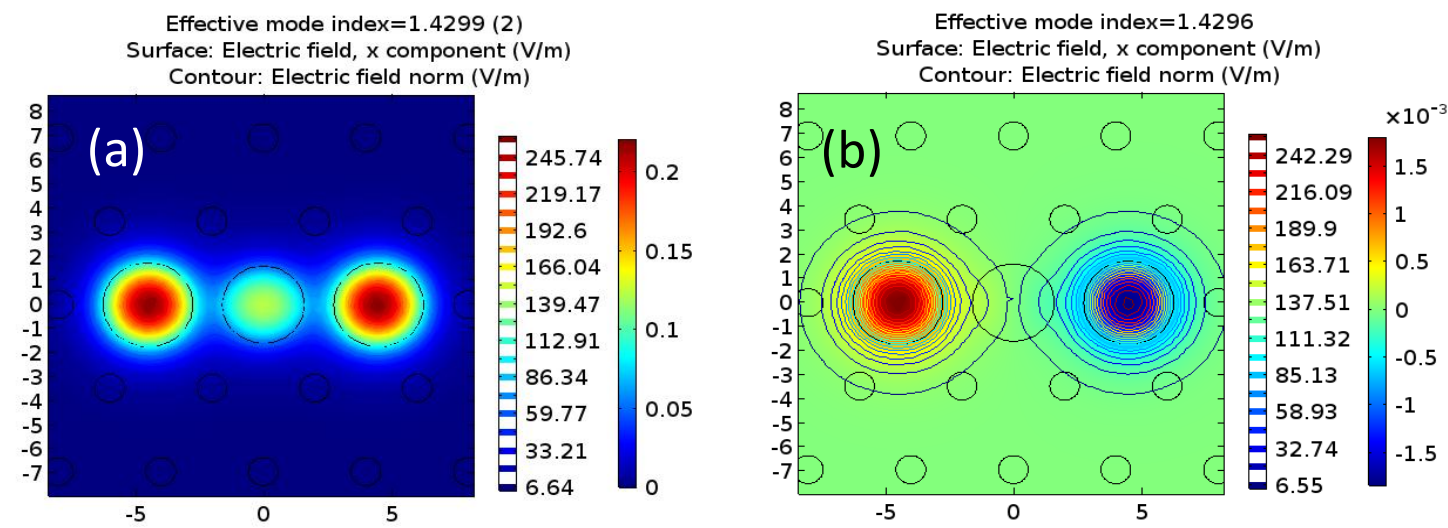

(c)
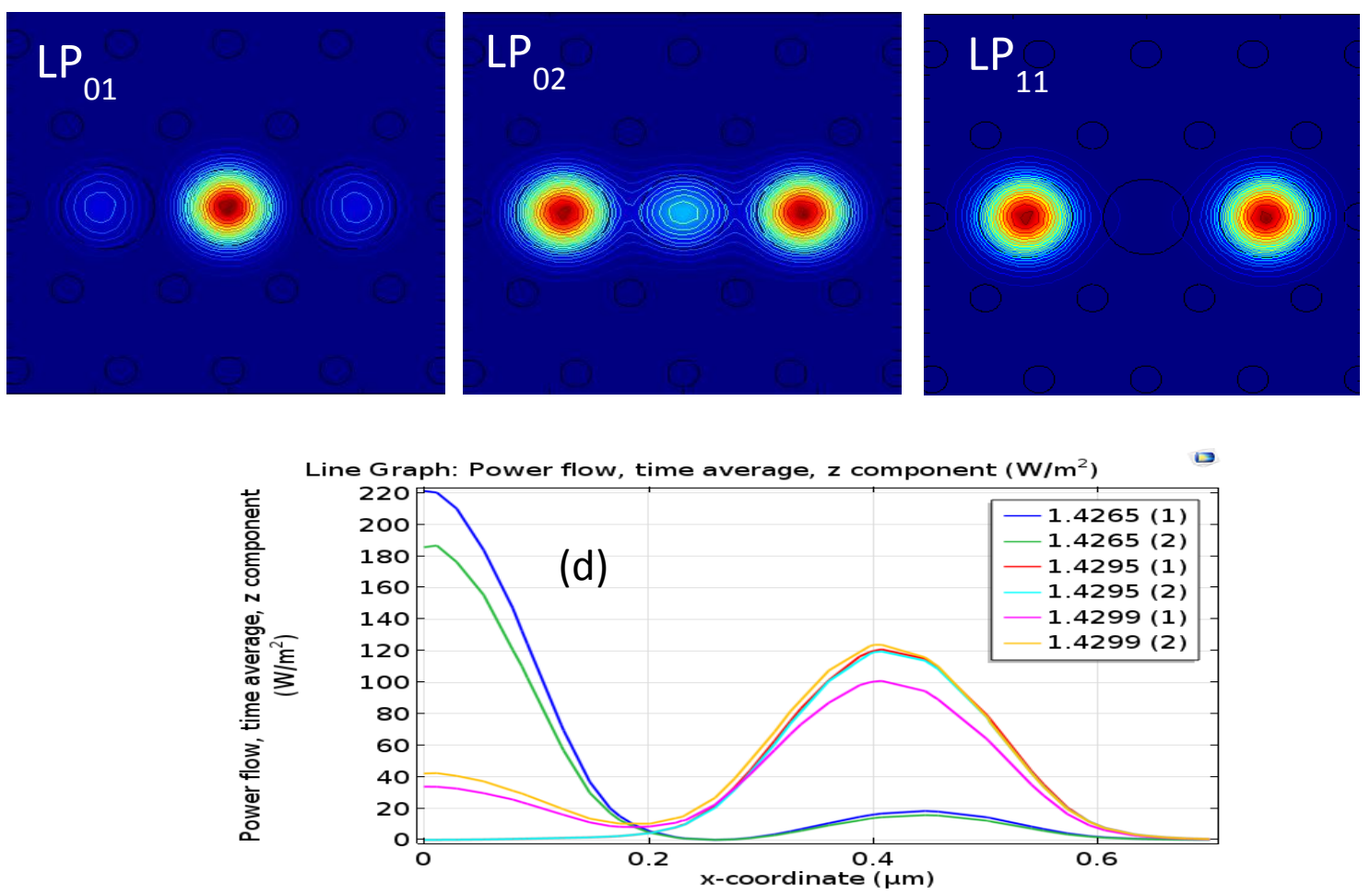

Fig. 4: Effective mode index and Surface: Electric field distributions of the $\mathrm{x}$ component for (a) Identical (b) Non-identical, three cores PCF coupler. (c) Power flow distributions of the six fundamental modes of the identical three-core PCF for core diameter of $3.2 \mu \mathrm{m}$. (d) Power flow for the fundamental modes of the three cores at wavelength $1.55 \mu \mathrm{m}$. 

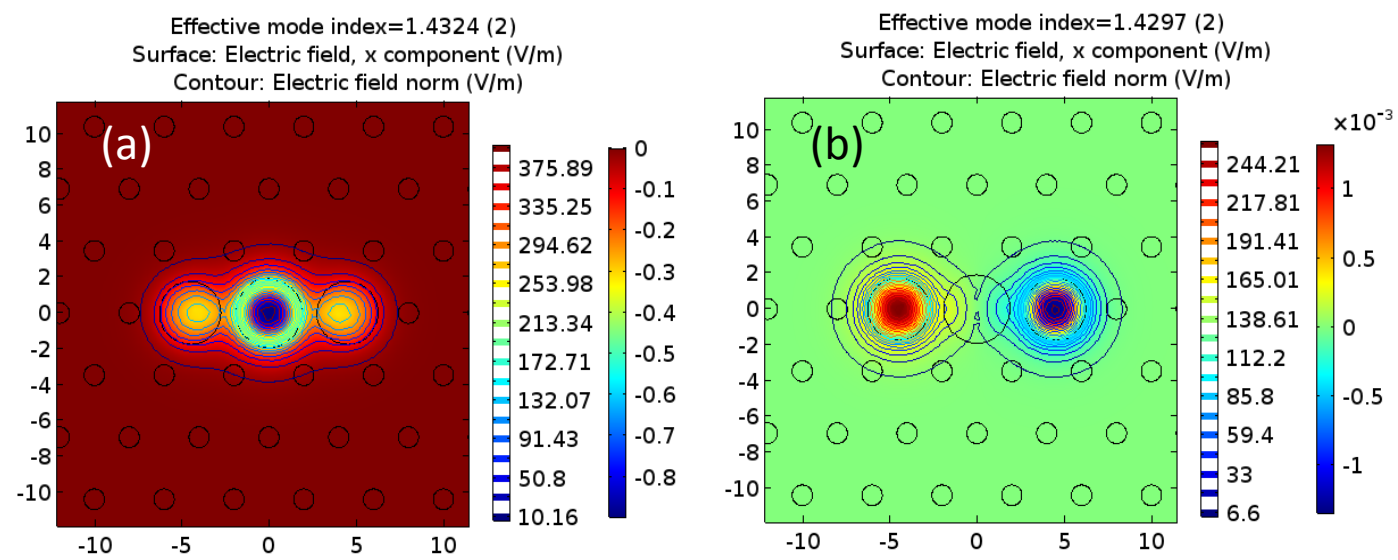

(c)
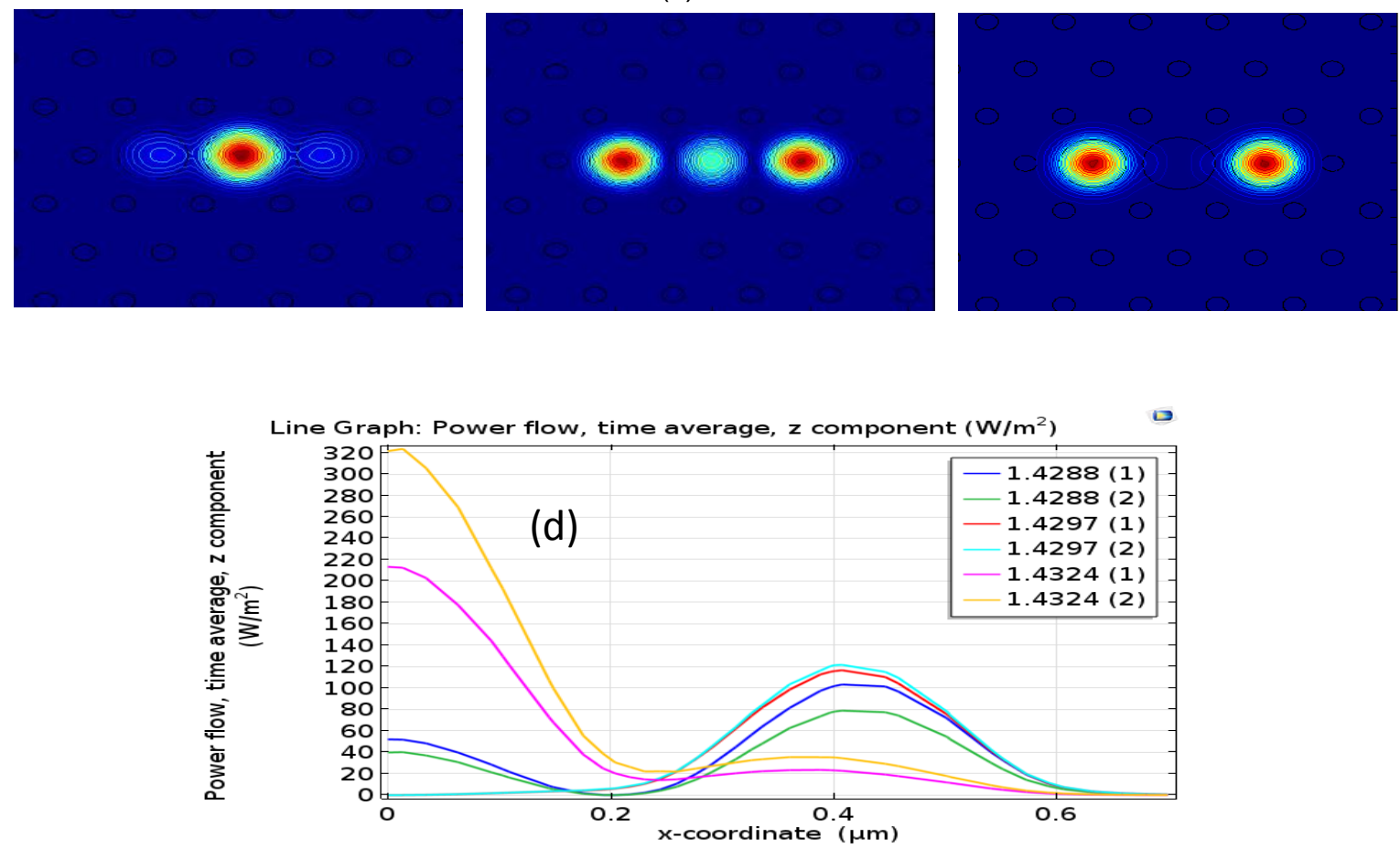

Fig. 5: Effective mode index and Surface: Electric field distributions of the $x$ component for (a) Identical (b) Non-identical, three cores PCF coupler for even modes. (c) Power flow distributions of the six fundamental modes of the identical three-core PCF by core diameter of $3.8 \mu \mathrm{m}$. (d) Power flow for the fundamental modes of the three cores. 
(a)
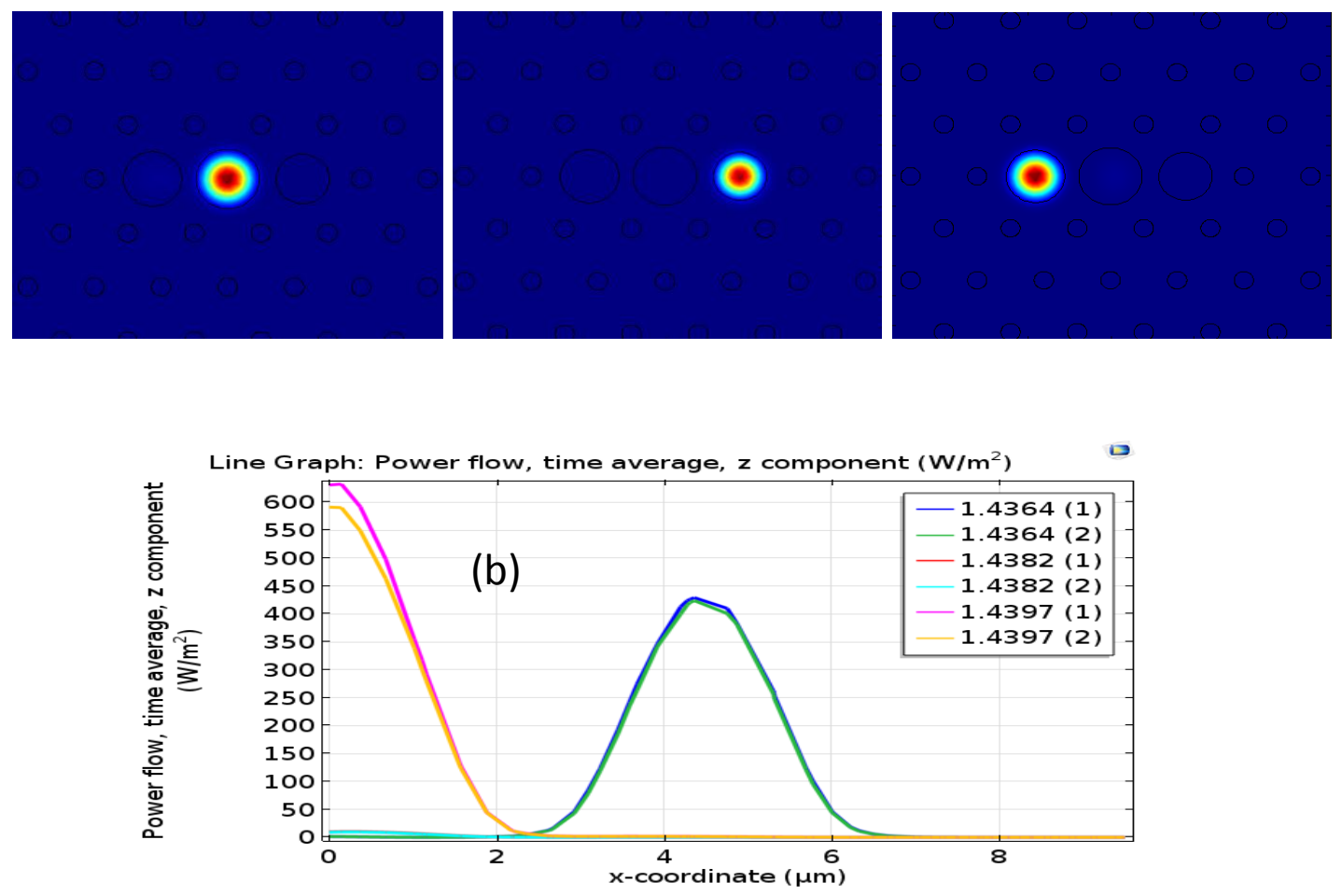

(c)
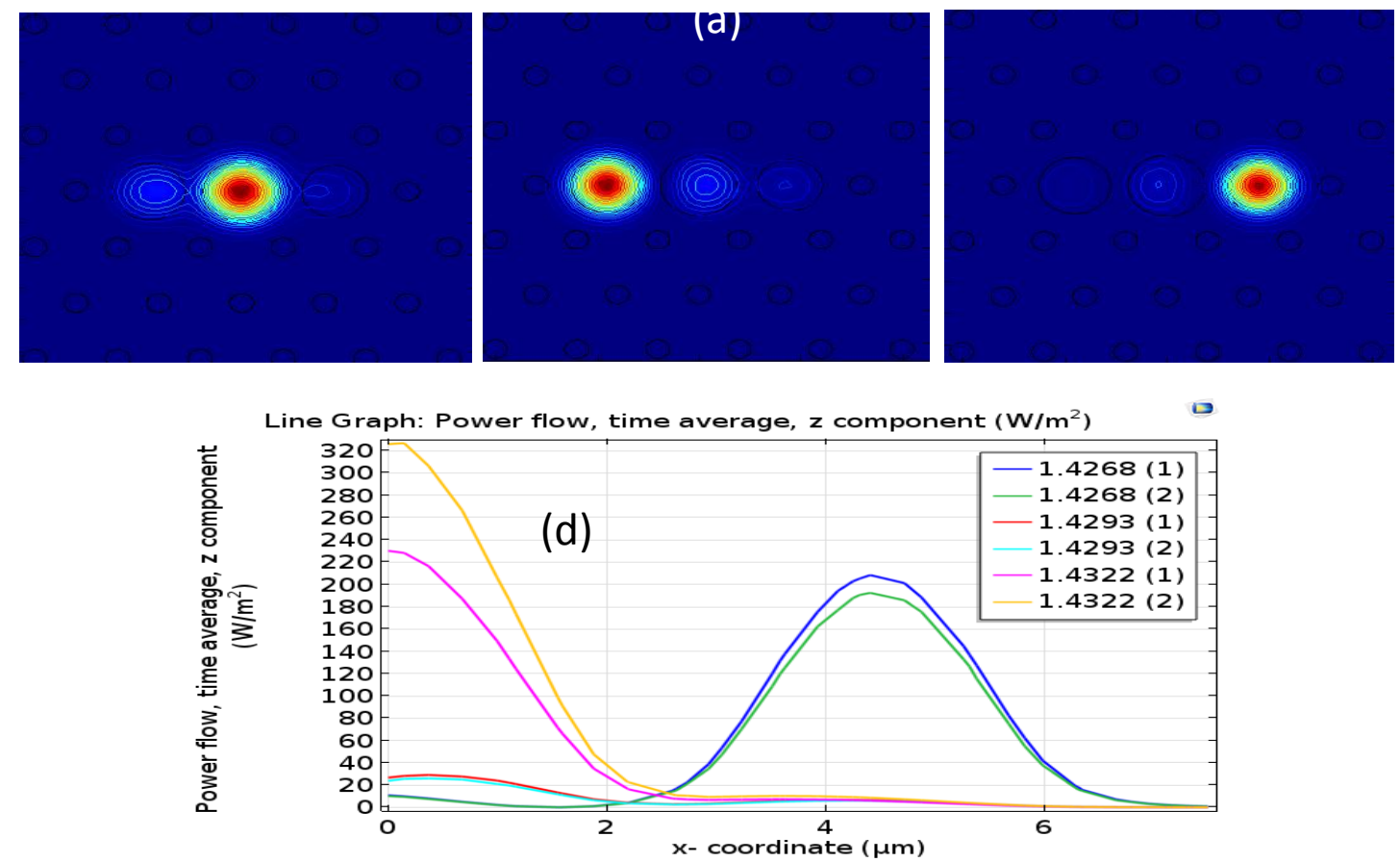

Fig. 6: The energy distributions between the three cores (a and b) at the wavelength of $1.064 \mu \mathrm{m}$. (c and d) at the wavelength of $1.55 \mu \mathrm{m}$.

To evaluate the effect of anisotropy in all core diameters on the coupling properties, we designed PCF coupler consisting of variation in all core diameters, such as $3.5 \mu \mathrm{m}, 3.2 \mu \mathrm{m}$, and 
$3.8 \mu \mathrm{m}$, with the same as the previous structural parameters at the wavelengths $1064 \mathrm{~nm}$ to analyze the extent of its coupling properties. The numerical results in Fig. (6 a) showed that the unique three power distributions with the refractive indices are 1.4364, 1.4382 and 1.4397 and the cores are decoupled with their neighbors and remain isolated in their location at the wavelength $1.064 \mathrm{~nm}$. As a result, the difference between core diameters will generate a small mismatch between the modes of these cores and prevent the coupling between them, i.e. the mode is isolated in a certain region in the array of the PCF. Fig. (6 b) represents an evaluation of Fig. 6(a), where each core has two modes, i.e., six modes of three cores become, we observe the two modes of the central core that is representing by both yellow and pink lines, and the two modes of the adjacent core to the central core represented by the blue and green colors, as well as the other adjacent core to the central core also has two modes light blue and red. All three patterns of hearts we see through the shape are separate and completely, not coupled with each other.

By increasing the wavelength to $1550 \mathrm{~nm}$ as shown in Fig. (6 c and d), we found through our numerical results the appearance of some coupling between cores of the three power distributions, i.e. the energy distributions with the refractive indices are $1.4268,1.4293$ and 1.4322 of the three cores are not unique and are some coupling between them as shown in Fig. (6 c). Fig. (6 d) represents an evaluation of Fig. $(6 \mathrm{c})$, where also, as we mentioned earlier, each of the three cores contains two modes, i.e., we get six modes, we notice that two modes of the central core represented by the yellow and pink lines with a weak coupling of two modes of the one of adjacent cores that are represented by the light blue and red colors, or we may see the two modes of the other adjacent core of the central core which are represented by the colors of the blue and green lines, it propagates separately from the modes of the other cores and is not completely coupling with them, or perhaps we may find a weak association between the patterns of each of the three cores. From our result we conclude that the coupling efficiency is a function of the wavelength, where the coupling efficiency increases as the wavelength increased; this study is useful in multiplexers and de-multiplexers applications.

\section{CONCLUSION}

We performed numerical simulations using COMSOL Multiphysics 5.5, for three-core PCFs with either identical or non-identical cores to show how the coupling properties between cores is affected when we induce a small change in the diameter of the central core relative to the outer cores. The coupling length, the coupling efficiency and the power transmission between cores are calculated. The results showed that the coupling exists between the identical cores. While the coupling is suppressed or strengthened between non-identical cores. We introduced anisotropy in all core diameters to introduce small mismatches between neighboring cores, the results found that some coupling occurs between the cores at a wavelength of $1550 \mathrm{~nm}$ and suppressed at the wavelength of $1064 \mathrm{~nm}$, this study is useful for multiplexing or demultiplexing applications.

This result can be therefore useful for space-division-multiplexed (SDM) optical transmission that is operating either without any coupling between modes or when the coupling exists between these modes; this means zero or small differential group delay (DGD). In the case of no coupling between modes, the modes can then be propagated independently and become separate modes while by presenting coupling between modes of the core, then mixed and can be separated by using multiple-input-multiple-output (MIMO) based digital signal processing (DSP) technique, this evidence super modes directly used as multiplexing or demultiplexing.

\section{ACKNOWLEDGEMENT}

This work was partially supported by the Republic of Iraq Ministry of High Education and Scientific Research scholarship (MoHESR Grant No. 16408). Also, the authors acknowledge the University of Muenster, department of physics, Germany for support. COMSOL Multiphysics ver. 


\section{REFERENCES}

Ahmad, A. K.; Khalifa, Z. (2020). Bio-sensing simulations using HC-PCF filled with different bioliquids. AIP Conference Proceedings 2213, 020131. https://doi.org/10.1063/5.0000121

Jollivet, C.; Mafi, A.; Flamm, D.; Duparré, M.; Schuster, K.; Grimm, S.; Schülzgen. A. (2014). Mode-resolved gain analysis and lasing in multi-super mode. J. Optical. Expres., 22, 30377- 30386.

Kishi, E.Y.N.; Kawabata, H. (1989). Modal and coupling field analysis of optical fibers with linearly distributed multiple cores. Lightwave Technology, 6(7), 902-907.

Li, P.; Zhao, J.; Zhang, X. (2010). Nonlinear coupling in triangular triple-core photonic crystal fibres. J. Optical. Expres., 18, 26828-6833.

Mohammed, M. (2019). Fem analysis of Two-Core photonic crystal fibre coupling characteristics. in Proc. CMES 2019, Kazimierz Dolny, Poland. www.biblioteka.pollub.pl/wydawnictwa

Mrejen, M.; Suchowski, H.; Hatakeyama, T.; Wu, C.; Feng, L.; O’Brien, K.; Wang, Y.; Zhang, X. (2015). Adiabatic elimination-based coupling control in densely packed subwavelength waveguides. J. Natural Comm., 8565, 1-7.

Parto, M.; Eftekhar, M. A.; Ali Miri, M.; Amezcua-Correa, R.; Li, G.; Christodoulides, D. N. (2016). Systematic approach for designing zero-DGD coupled multi-core optical fibres. $J$. Optical. Lett., 41, 1917-1920.

Reichenbach, K. L.; Xu, C. (2007). Numerical analysis of light propagation in image fibers or coherent fiber bundles. Optics Express, 15(5), 2151-2165.

Reichenbach, K. L.; Xu, C. (2005). Independent core propagation in two-core photonic crystal fibers resulting from structural nonuniformities. Optics Express, 13(25), 10336-10348.

Szostklewicz, L.; Napierala, M.; Ziolowicz, A.; Pytel, A.; Tenderenda, T.; Nasilowski, T. (2016). Cross talk analysis in multicore optical fibres by supermode theory. J. Optical. Lett., 41, 3759-3762.

Tan, R.Z.; Ren, G. (2015). Analytical formulation of supermodes in multicore fibres with hexagonally distributed cores. J. Photon. Soc., 7, 7100311.

Wu, Y.; Chiang, K. (2015). Compact three-core fibres with ultra-low differential group delays for broadband mode division multiplexing. J. Optical. Expres., 23, 20867-20875.

Yan, Y.; Toulouse, J.; Velchev, I.; Rotkin, S. V. (2008). Decoupling and Asymmetric Coupling in a Triple-core Photonic Crystal Fibre (PCFs). J. Optical. Soc. Am., B25, 1488- 1495.

Yu, X.; Liu, M.; Chang, Y.; Yan M.; Shum, P. (2006). Coupling coefficient of two-core microstructured optical fiber. Optics Communications, 260, 164-169. https://doi.org/10.1016/j.optcom.2005.10.040 


\section{تصميم فايير بلوري ضوئي متعدد النوى لتطبيقات تعدد الارسال}

\section{(Multiplexers and Demultiplexers)}

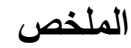

تتأثر خصائص الاقتران بشكل كبير عندما نكون النوى الثناثة من الألياف البلورية الضوئية منطابقة أو غير منطابقة، نقوم بتحليل هذا عدديا باستخدام برنامج 5.5 COMSOL Multiphysics للحصول على نظرة ثاقبة لطبيعة الاقتران بين هذه النوى. تتتج النوى غير المتطابقة من خلا عدم تطابق طفيف في ثوابت الانتشار وهي كافية لقمع الاقتران بين النوى أو ربما تقوية

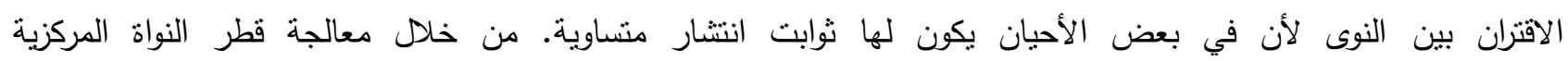

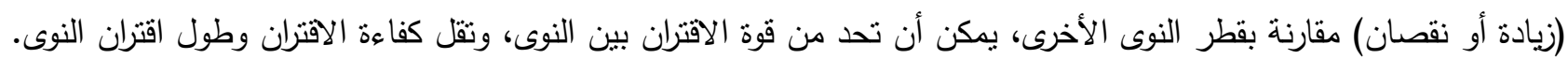

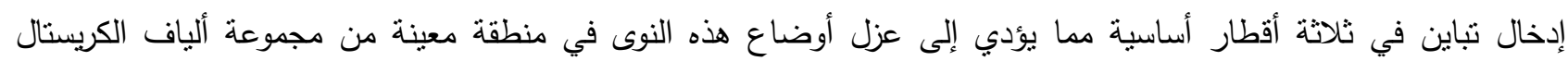

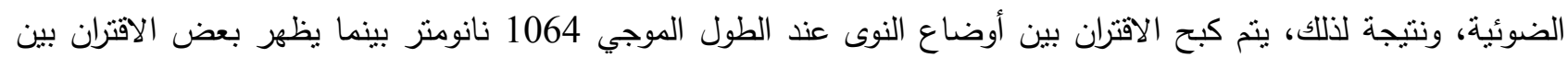

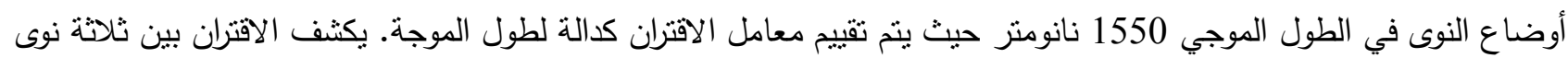
عن خصائص جديدة أكثر من نوى وهذا مفيد في تطبيقات تعدد الإرسال. الكلمات الدالة: الألياف البلورية الضوئية، تصميم الألياف، طول الاقتران، قوة الاقتران، تعدد الارسال. 